\title{
Efficacy of Catheter-directed Thrombolysis on Post-burn Deep Venous Thrombosis of Lower Extremity
}

\author{
Guozheng Gao1, Shugang Zhu², Zhen Xie', Jiasen Wang3 and Baohong Yao3
}

\begin{abstract}
Objective: To observe the clinical effect of catheter-directed thrombolysis on deep venous thrombosis (DVT) of lower extremity after burn.

Study Design: An observational study.

Place and Duration of Study: Invasive Technology Department, Binzhou City Center Hospital, China, from January 2015 to November 2017.

Methodology: Eighty-two patients with lower extremity DVT after burn were selected as the study object. All patients received catheter-directed thrombolysis and the clinical efficacy was evaluated. The blood coagulation parameters including plasma prothrombin time (PT), activated partial thromboplastin time (APTT), thrombin time (TT), and fibrin original (FIB) and inflammatory response factors including interleukin (IL)-6, C-reactive protein (CRP) and D-dimer were compared before and after treatment.

Results: All interventional surgeries that the patients received were successful. The effective rate of catheter-directed thrombolysis was $98.78 \%$ (81 cases). After 6 days of treatment, compared with that before treatment, serum PT, APTT and TT were up-regulated (all $p<0.001)$ and FIB was down-regulated $(p<0.001)$. After 6 days of treatment, the levels of serum IL-6, CRP and D-dimer were lower than those before treatment (all $p<0.001)$. No visceral hemorrhage occurred after 1 week of treatment.

Conclusion: Catheter-directed thrombolysis of patients with lower extremity DVT after burn produces a good effect. It can improve the coagulation function and reduce the level of inflammatory response factors in patients with high safety.
\end{abstract}

Key Words: Burn, Lower extremities, Deep vein thrombosis (DVT), Catheter-directed thrombolysis.

\section{INTRODUCTION}

Lower extremity deep vein thrombosis (DVT) is a common complication in trauma patients. ${ }^{1}$ With the progression of lower extremity DVT disease, serious complications such as lower limb soft tissue necrosis, pulmonary embolism and cerebral embolism may occur. Lower extremity DVT has a high disability rate and may lead to death in severe cases. ${ }^{2}$ The formation of lower extremity DVT in burned patients is the result of multiple factors which interact with each other. ${ }^{3}$ For severely burned patients, due to surgical trauma, long-term bedridden status, repeated intravenous puncture and deep venous catheterization, especially nutrition support and long-term infusion through the femoral vein and inferior vena cava intubation, their vascular inflammation caused by bacterial and viral infections, as well as other factors, can initiate the exogenous, endogenous coagulation

1 Department of Invasive Technology, Binzhou City Center Hospital, 251700, China

2 Department of Burn / Emergency ${ }^{3}$, Yantai Affiliated Hospital of Binzhou Medical University, 264100, China

4 Department of Thoracic Surgery, Binzhou Medical University Hospital, 256603, China

Correspondence: Dr. Zhen Xie, Department of Thoracic Surgery, Binzhou Medical University Hospital, 256603, China E-mail:jdwwk5@163.com

Received: July 03, 2018; Accepted: November 26, 2018 process, thereby increasing the possibility of venous thrombosis. 4 In the past, intravenous thrombolytic agents or surgeries were mainly used for the treatment of patients with lower extremity DVT.5,6 However, the efficacy was poor, and it could increase bleeding risk. Surgical treatment also suffered larger wounds. In recent years, catheter-directed thrombolysis has attracted more and more attention in clinical practice. This therapy has the advantages of minimal invasiveness, high efficiency, and short hospital stay. Patients are willing to accept it. Previous studies had shown that catheter-directed thrombolysis approach was a feasible, effective, and safe method for acute extensive lower extremity DVT patients. ${ }^{7}$ However, catheter-directed thrombolysis is not suitable for all patients with proximal DVT. ${ }^{8}$ At present, there are few reports about catheterdirected thrombolysis in the treatment of lower extremity DVT after burn.

The objective of this study was to investigate the clinical efficacy of catheter-directed thrombolysis of patients with lower extremity DVT after burn, in order to provide important reference for the catheter-directed thrombolysis applied in the field of burns.

\section{METHODOLOGY}

This study was conducted at Invasive Technology Department, Binzhou City Center Hospital, China, from January 2015 to November 2017 and approved by the 
Hospital Ethical and Research Committee. A total of 82 patients with DVT of lower extremity after burn treatment were selected. Inclusion criteria were: First-episode DVT patients and course of disease was less than 21 days; patients were diagnosed with DVT by color ultrasound; unilateral lower extremity burns; unilateral limb pain and swelling; without fractures, dislocations or other organ injuries; blood D-dimer greater than $500 \mathrm{ng} / \mathrm{L}$. Exclusion criteria were: Patients with severe cardiopulmonary insufficiency; impaired liver function; dangerously high blood pressure. Contraindications for patients included anticoagulation and thrombolytic therapy; patients with cancer; patients with a history of allergy; or previous reaction to contrast media.

The patient was placed in a supine position, and puncture approach through the right internal jugular vein or contralateral femoral vein was selected. After successful puncture, the $5 \mathrm{~F}$ pigtail catheter was placed. The tip of the catheter was placed in the iliac vein of the healthy side and the entire inferior vena cava was used for imaging to understand its morphology, presence of false lumen or not, intraluminal thrombosis, anatomic variations, and patency. After the position of the opening of the renal vein was determined, a temporary/ permanent dual-use Gunther Tulip inferior vena cava filter (IVCF) was placed below the lower margin of the renal vein orifice. If there was a thrombus in the inferior vena cava at or below the renal vein level, the IVCF could be placed above the opening of the renal vein. UniFuse thrombolytic catheter was retrogradely inserted into the deep vein of the affected limb with the help of guide wire and the catheter was kept (Figure 1). The catheter perfusion segment fully covered the iliac and femoral venous thrombosis. Intraoperative catheterpulsed injection of $300,000 \mathrm{U}$ urokinase and postoperative micro-pump continuous injection of urokinase $(600,000 \mathrm{U} / \mathrm{d})$ were performed. The catheter was retained for not more than 7 days.

Ultrasound or contrast examination of the veins of lower extremity and inferior vena cava prior to IVCF removal was performed to assess the risk. IVCF removal criteria were the time after IVCF implantation did not exceed the time limit specified in the instructions. The angiography confirmed that there was no free floating thrombus or fresh thrombus in the popliteal, femoral, iliac veins and inferior vena cava, or the above thrombus disappeared after treatment. The IVCF was removed from the filter via a $10 \mathrm{~F}$ recycling sheath after the femoral venous access to the end of the filter was captured using a gooseneck snare.

Intraoperative routine indwelling thrombolysis catheter was conducted, except for the following four situations: Puncture point was too close to the burn wound (distance $<3 \mathrm{~cm}$ ); puncture point passed through the recent (within 1 week) skin removal or graft surgery area; the patients were in critical condition, especially complicated with hypovolemic shock, septic shock, etc.; and the patients showed bleeding tendency, especially the presence of thrombolytic contraindications.

After 6 days of treatment, the clinical efficacy was assessed as excellent, good, fair and poor, according to the patient's postoperative interventional physical examination and angiography results. The criteria for excellence were the circumference, tension, and activity of the affected limb after the treatment were basically normal, and difference in circumference was less than or equal to $1.0 \mathrm{~cm}$; angiography showed that full or basic recovery of blood flow; the abnormal collateral vessels did not appear; there were no remains of contrast agent, and the wall of the tube was smooth. The criteria for good were the circumference, tension, and activity of the affected limb were close to normal, and difference in circumference was $1.0-1.5 \mathrm{~cm}$; the angiography showed that most of the blood flow restored, and there was a small amount of collateral vessels; there were no obvious remains of contrast agent, and the vessel wall was smooth. The criteria for fair were the circumference, tension, and activity of the affected limb were significantly improved, and difference in circumference was 1.5 to $2.0 \mathrm{~cm}$; the angiography showed that the blood flow was partly restored, and there were more collateral vessels. There were slight remains of the contrast agent, and the vessel wall was less smooth. The criteria for poor were that there were no significant improvements in circumference, tension, and activity of the affected limb, and the differences in circumference was $>2.0 \mathrm{~cm}$; the angiography showed no recovery of blood flow, and there were a large number of collateral vessels; there were a lot of remains of contrast agent, and the wall was not smooth. The ratings of excellent, good, and fair were considered effective.

Venous blood samples were collected before and 6 days after treatment. Serum coagulation parameters including plasma prothrombin time (PT), activated partial thromboplastin time (APTT), thrombin time (TT) and fibrin original (FIB) were measured with a blood coagulation analyser. Serum inflammatory response factors including interleukin (IL)-6, C-reactive protein (CRP) and D-dimer were measured by enzyme-linked immunosorbent assay. The occurrence of bleeds, such as gingival bleeding, skin ecchymosis, cerebral hemorrhage, etc., was observed and recorded after one week of treatment.

SPSS version 22.0 software was adopted for data statistical analysis. Continuous variables were represented as mean $\pm S D$, examined by paired t-tests. Categorical data were represented by number $(n)$ and percentage (\%). A $p$-value of less than $5 \%$ were regarded as significant.

\section{RESULTS}

Among the subjects, there were 42 males $(51.22 \%)$ and 40 females (48.78\%); they were aged $39-68$ years, with mean age of $53.24 \pm 3.57$ years. The burn area was 
Table I: Comparison of coagulation parameters before and after 6 days of treatment.

\begin{tabular}{|c|c|c|c|c|c|c|c|c|c|}
\hline \multirow[t]{2}{*}{ Time } & \multirow[t]{2}{*}{$\mathrm{n}$} & \multicolumn{2}{|c|}{ PT (s) } & \multicolumn{2}{|c|}{ APTT (s) } & \multicolumn{2}{|c|}{$\mathrm{TT}(\mathrm{s})$} & \multicolumn{2}{|c|}{$\mathrm{FIB}(\mathrm{g} / \mathrm{L})$} \\
\hline & & Mean \pm SD & $p$-value & Mean \pm SD & p-value & Mean \pm SD & p-value & Mean \pm SD & p-value \\
\hline Before treatment & 82 & $9.78 \pm 1.89$ & $<0.001$ & $31.92 \pm 1.91$ & $<0.001$ & $12.06 \pm 1.30$ & $<0.001$ & $5.19 \pm 1.45$ & $<0.001$ \\
\hline After 6 days of treatment & 82 & $18.41 \pm 2.19$ & & $38.63 \pm 3.98$ & & $17.88 \pm 1.77$ & & $3.93 \pm 0.59$ & \\
\hline
\end{tabular}

Table II: Comparison of inflammatory response factors before and after 6 days of treatment.

\begin{tabular}{|c|c|c|c|c|c|c|c|}
\hline \multirow[t]{2}{*}{ Time } & \multirow[t]{2}{*}{$n$} & \multicolumn{2}{|c|}{ IL-6 (pg/mL) } & \multicolumn{2}{|c|}{ CRP (mg/L) } & \multicolumn{2}{|c|}{ D-dimer (mg/L) } \\
\hline & & Mean \pm SD & $p$-value & Mean \pm SD & $p$-value & Mean $\pm S D$ & $\mathrm{p}$-value \\
\hline Before treatment & 82 & $27.15 \pm 3.81$ & $<0.001$ & $31.26 \pm 4.69$ & $<0.001$ & $5.28 \pm 1.14$ & $<0.001$ \\
\hline After 6 days of treatment & 82 & $9.16 \pm 1.34$ & & $4.53 \pm 0.56$ & & $2.47 \pm 0.58$ & \\
\hline
\end{tabular}

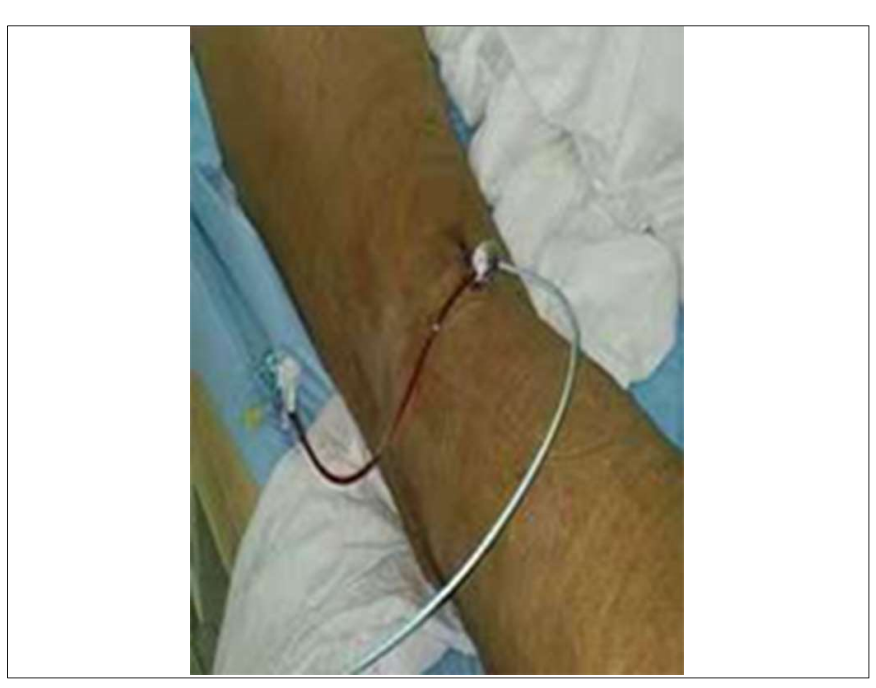

Figure 1: Catheter thrombolysis retention after popliteal vein puncture.

$1-86 \%$, the average being $36.85 \pm 12.33 \%$. DVT was found at 12 to 70 days after burn, with an average of $39.16 \pm 9.65$ days.

All patients were successfully treated with interventional surgeries and received IVCF and anticoagulant therapy. Sixty-seven $(81.71 \%)$ cases of contact thrombolysis through indwelled thrombolytic catheters, 12 (14.63\%) cases of intravenous thrombolysis, $3(3.66 \%)$ cases without thrombolysis; and no symptomatic pulmonary embolism (PE) occurred. The efficacy evaluation was excellent in 51 cases $(62.20 \%)$, good in 20 cases $(24.39 \%)$, fair in 10 cases $(12.20 \%)$, and poor in 1 case $(1.22 \%)$. The effective rate of catheter-directed thrombolysis was $98.78 \%$ (81 cases). Compared with those before treatment, serum PT, APTT and TT were upregulated after 6 days of treatment (all $p<0.001$ ), and FIB was down-regulated ( $p<0.001$, Table I). After 6 days of treatment, the levels of serum IL-6, CRP and D-dimer were lower than those before treatment (all $p<0.001$, Table II). No visceral hemorrhage occurred after one week of treatment.

\section{DISCUSSION}

In recent years, interventional therapies have been gradually used to treat deep vein thrombosis of lower extremity. 9 To insert thrombolytic catheter into the thrombus and achieve contact thrombolysis is the key to catheter-directed thrombolysis of DVT of the lower extremity. ${ }^{10,11}$ Prior to contacting thrombolysis, routine placement of IVCF is an ideal measure for preventing fatal pulmonary embolism (PE). IVCF not only prevents pulmonary embolism (PE), but also minimises the mortality of patients with lower extremity DVT.12,13 Enden et al. have shown that anticoagulation combined with contact thrombolysis in the treatment of deep vein thrombosis of the lower extremity can significantly improve the patency of the iliac femoral vein and reduce the incidence of postthrombotic syndrome and bleeding complications. ${ }^{14}$ This study found that compared with that before treatment, serum PT, APTT and TT were up-regulated and FIB was down-regulated after 6 days of treatment. It suggested that catheter-directed thrombolysis could effectively control the patients, and improve the hypercoagulable state of the patient's blood.

Roumen-Klappe et al. found that serum CRP, interleukin and other inflammatory factors in patients with acute lower extremity DVT were significantly higher, indicating that inflammatory factors may play an important role in the formation of acute lower extremity DVT.15 Fox et al. found that the serum CRP level in patients with acute venous thrombosis could be increased by 2-6 times, indicating that the organism's inflammatory response was activated during acute DVT. 16 Thrombosis activates multiple inflammatory pathways and stimulates high expression of inflammatory factors, including IL-6 and CRP. In addition, animal experiments had confirmed that the peak concentration of IL-6 occurs 2 days after the formation of DVT and is related to the size of the thrombus. ${ }^{17} \mathrm{D}$-dimer is a specific degradation product produced via the hydrolysis of plasmin after fibrin monomer is cross-linked by activating factors, and is a molecular marker reflecting the hypercoagulable state and activation of fibrinolysis in vivo. When blood clotting and secondary fibrinolysis occurs in the body, it will appear in blood in large quantities. Therefore, D-dimer can be used as one of the molecular markers of hypercoagulability and hyperfibrinolysis in vivo. Qualitative or quantitative detection of D-dimer in plasma has early diagnostic value for thrombotic diseases. ${ }^{18,19}$ The study 
found that after 6 days of treatment, the levels of serum IL-6, CRP and D-dimer were lower than those before treatment. It suggested that catheter-directed thrombolysis could reduce the level of inflammatory response factors in patients.

This study demonstrated that catheter-directed thrombolysis of lower extremity DVT after burn had good clinical efficacy. There was no symptomatic pulmonary embolism (PE). All patients had no organ bleeding for a week with higher safety. However, the treatment of DVT after burn was particular, and it needs to be further explored in the following aspects: First, selection of puncture points. The right internal jugular vein or contralateral femoral vein was often chosen for the puncture approach of DVT catheter-directed thrombolysis. In this group of patients, $63.41 \%$ (52 cases) had a burn area over $30 \%$. The proposed puncture area was often covered with burn wound or skin grafting or skin wound after removal. And the burn wound itself was accompanied by bacterial infection, so the selection of puncture point was limited compared with that of the non-burn patients. So for such cases, the contralateral femoral vein puncture should be selected, mainly because there was wound burn in the right internal jugular vein puncture area and it was difficult for the patient to fit with the puncture position. At the same time, it is recommended that the puncture point be selected to be more than $3 \mathrm{~cm}$ away from the burn wound, without going through the recent skin removal or grafting surgical area. In order to avoid the possible consequences of catheter-related infection, puncture access to burn wounds should not be selected. Second, whether to indwell thrombolysis catheter or not. Catheter-directed thrombolysis therapy is to place the thrombolysis catheter in the venous thrombosis and let the thrombolytic agents directly act on the thrombus. It can significantly improve the thrombolytic rate. With efficacy better than intravenous thrombolysis, it is the current important means for catheter-directed thrombolysis of DVT.20 In this study, 15 cases (18.29\%) had no routine indwelling contact thrombolysis catheter; the reason of which was related to the burn area, location and condition. Among them, 12 cases $(14.63 \%)$ were cancelled to avoid related infection since the puncture site was too close to the burn wound or skin surgery area. Three cases $(3.66 \%)$ were cancelled to avoid catheter-related infection, bleeding, and due to serious illness.

\section{CONCLUSION}

In short, catheter-directed thrombolysis of patients with lower extremity DVT, after burn, produces a good effect. It can improve the coagulation function and reduce the level of inflammatory response factors in patients with high safety. It is worth mentioning that, compared with the non-burn DVT intervention, the catheter-directed thrombolysis of burn DVT has its particularity in terms of selection of puncture site, whether to retain the thrombolytic catheter or not, etc. As such, it needs to be further studied in the future clinical practice.

\section{REFERENCES}

1. Bawa H, Weick JW, Dirschl DR, Luu HH. Trends in deep vein thrombosis prophylaxis and deep vein thrombosis rates after total hip and knee arthroplasty. J Am Acad Orthop Surg 2018; 26:698-705.

2. Shaikhouni A, Baum J, Lonser RR. Deep vein thrombosis prophylaxis in the neurosurgical patient. Neurosurg Clin N Am 2018; 29:567-74.

3. Shirol SS, Kodaganur S, Rao MR, Tiwari V. The conundrum of deep vein thrombosis prophylaxis in burns in India and review of literature. Indian J Plast Surg 2017; 50:288-94.

4. Sikora S, Papp A. Venous thromboembolism in burn patients is not prevented by chemoprophylaxis. Burns 2017; 43:1330-4.

5. Fleck D, Albadawi H, Shamoun F, Knuttinen G, Naidu S, Oklu R. Catheter-directed thrombolysis of deep vein thrombosis: literature review and practice considerations. Cardiovasc Diagn Ther 2017; 7(Suppl 3):S228-37.

6. Zaghlool DS, Franz RW, Jenkins J. Eko Sonic Thrombolysis as a therapeutic adjunct in venous occlusive disease. Int J Angiol 2016; 25:203-9.

7. Wang $H$, Qi X, Luo H, Zhang Q, Chen $Y$, Sun J. Catheterdirected thrombolysis through anterior tibial vein for treating acute extensive deep venous thrombosis. J Vasc Surg Venous Lymphat Disord 2018; 6:681-8.

8. Chiasakul T, Cuker A. The case for catheter-directed thrombolysis in selected patients with acute proximal deep vein thrombosis. Blood Adv 2018; 2:1799-802.

9. Qiang L, Yu Z, Wang J, Xiao C, Lin L. Long-term prognostic analysis of early interventional therapy for lower extremity deep venous thrombosis. Exp Ther Med 2016; 12:3545-8.

10. Kwon SH, Oh JH, Seo TS, Ahn HJ, Park HC. Percutaneous aspiration thrombectomy for the treatment of acute lower extremity deep vein thrombosis: Is thrombolysis needed? Clin Radiol 2009; 64:484-90.

11. Elbasty A, Metcalf J. Safety and efficacy of catheter direct thrombolysis in management of acute iliofemoral deep vein thrombosis: A systematic review. Vasc Specialist Int 2017; 33:121-34.

12. Son J, Bae M, Chung SW, Lee CW, Huh U, Song S. Should we remove the retrievable cook celect inferior vena cava filter? Eight years of experience at a single center. Korean $J$ Thorac Cardiovasc Surg 2017; 50:443-7.

13. Brunson A, Ho G, White R, Wun T. Inferior vena cava filters in patients with cancer and venous thromboembolism (VTE) does not improve clinical outcomes: A population-based study. Thromb Res 2017; 153:57-64.

14. Enden T, Haig Y, Kløw NE, Slagsvold CE, Sandvik L, Ghanima W, et al. Long-term outcome after additional catheter-directed thrombolysis versus standard treatment for acute iliofemoral deep vein thrombosis (the CaVen $\mathrm{T}$ study): A randomised controlled trial. Lancet 2012; 55:31-8.

15. Roumen-Klappe EM, den Heijer M, van Uum SH, van der VenJongekrijg J, van der Graaf F, Wollersheim H. Inflammatory 
response in the acute phase of deep vein thrombosis. $J$ Vasc Surg 2002; 35:701-6.

16. Fox EA, Kahn SR. The relationship between inflammation and venous thrombosis: A systematic review of clinical studies. Thromb Haemost 2005; 94:362-5.

17. Wakefield TW, Greenfield LJ, Rolfe MW, Strieter RM, Abrams GD, Kunkel SL, et al. Inflammatory and procoagulant mediator interactions in an experimental baboon model of venous thrombosis. Thromb Haemost 1993; 69:164-72.
18. Bates SM, Jaeschke R, Stevens SM, Goodacre S, Wells PS, Stevenson MD, et al. Diagnosis of DVT. Chest 2012; 141: e351S-e418S.

19. Ahmad A, Jamjute P, Ghosh T, Klazinga DA. D-dimer negative deep vein thrombosis in puerperium. Eur Clin Obstetr Gynaecol 2008; 3:131-4.

20. Shi Y, Shi W, Chen L, Gu J. A systematic review of ultrasoundaccelerated catheter-directed thrombolysis in the treatment of deep vein thrombosis. J Thromb Thrombolysis 2018; 45:440-51.

......... 